\title{
On conduction mechanism in paramagnetic phase of Gd based manganites
}

\author{
S SAGAR ${ }^{1,2}$ and M R ANANTHARAMAN ${ }^{1, *}$ \\ ${ }^{1}$ Department of Physics, Cochin University of Science and Technology, Cochin 682 022, India \\ ${ }^{2}$ Present address: Department of Physics, Govt. Polytechnic College, Adoor 691 523, India
}

MS received 19 September 2008; revised 31 January 2011

\begin{abstract}
Materials belonging to the family of manganites are technologically important since they exhibit colossal magneto resistance. A proper understanding of the transport properties is very vital in tailoring the properties. A heavy rare earth doped manganite like $\mathrm{Gd}_{0.7} \mathrm{Sr}_{0.3} \mathrm{MnO}_{3}$ is purported to be exhibiting unusual properties because of smaller ionic radius of $\mathrm{Gd}$. $\mathrm{Gd}_{0.7} \mathrm{Sr}_{0.3} \mathrm{MnO}_{3}$ is prepared by a wet solid state reaction method. The conduction mechanism in such a compound has been elucidated by subjecting the material to low temperature d.c. conductivity measurement. It has been found that the low band width material follows a variable range hopping (VRH) model followed by a small polaron hopping (SPH) model. The results are presented here.
\end{abstract}

Keywords. Manganite; conduction mechanism; small polaron hopping; variable range hopping.

\section{Introduction}

Manganites and cobaltites belong to the perovskite class of materials. They exhibit interesting magnetic, electrical and structural properties. This class of materials has been in the forefront of material research for three decades or so. Among them manganites occupy a special position because of its application potential as catalysts, as electrode materials and in various other applications like bolometer (Pedersen and Libby 1972; Goyal et al 1997; Venkatesan et al 1998; Coey and Viret 1999). Manganites are mixed valence manganese oxides. There is renewed interest in these classes of materials after the discovery of colossal magneto-resistance. They exhibit simultaneous phase transitions viz. from ferromagnetic to paramagnetic and metal to insulator. From a fundamental point of view the interplay of various factors that influence the electrical transport properties assume significance. A variety of parameters like charge, orbital coupling, magnetic ordering and lattice degrees of freedom have profound influence on the electrical and magnetic properties of these materials. From an application perspective they are ideal magneto-resistance sensors for biological applications as well as magnetic read heads in hard disk and other magnetic storage devices (Balcells et al 1996; Hwang et al 1996; Lawler et al 1996; Mahendiran et al 1996). It may be noted here that large colossal magnetoresistance (CMR) was first reported in hole-doped manganese oxide perovskites. Other compounds such as double perovskites, manganese oxide pyrochlores and europium hexaborides, among others, also exhibit the same striking property of manganites

\footnotetext{
*Author for correspondence (mraiyer@yahoo.com)
}

(Morimoto et al 1996; Coey and Viret 1999). In these compounds, a $\mathrm{Mn}^{3+} / \mathrm{Mn}^{4+}$ double exchange interaction reduces the transfer energy of the Mn $3 d$ holes through a parallel alignment of neighbouring Mn spins resulting in a CMR near the Curie temperature $\left(T_{\mathrm{c}}\right)$. However, further investigations revealed that the double exchange alone cannot quantitatively account for the observed CMR effect, and that the strong electron-phonon interaction arising out of Jahn-Teller splitting may also have to be taken into account (Pollert et al 1982; Zhao et al 1996). The strong Jahn-Teller effect due to $\mathrm{Mn}^{3+}$ leads to the formation of polarons; this strongly influences the transport properties. Other parameters that influence the magneto-resistance properties of manganites are chemical composition, radius of $A$ site cation, amount of hole doping $(x)$ and random disorder of the $A$ site. It has been known for decades that the magnetic phases and electronic properties of these manganites vary with the doping level $(x)$. Upon increasing $x$, the concentration of $\mathrm{Mn}^{4+}$ increases, giving rise to a mixture of $\mathrm{Mn}^{3+}$ and $\mathrm{Mn}^{4+}$ which initially yields canted spin configurations and then forms metallic bonding and subsequent ferromagnetism for doping levels lying in the range $0 \cdot 2-0 \cdot 4$. The occurrence of ferromagnetism is attributed to the double-exchange interaction between $\mathrm{Mn}^{3+}$ and $\mathrm{Mn}^{4+}$ ions. Metal to insulator (M-I) transition is observed in the case of lanthanum manganites at near room temperatures (Zener 1951). However, manganites doped with smaller rare earth ions like $\mathrm{Gd}, \mathrm{Pr}$ exhibited metal insulator transition only at very low temperatures. At the same time when the M-I transition is attained for low average radius $A$-site cation perovskites, higher magnetoresistance ratio is obtained. This makes this heavy rare earth based manganites very attractive for further investigation (Garcia-Landa et al 1998). Relatively, 
fewer studies are reported on perovskites based on heavy rare earths. This is because it was presumed that smaller radius cation in the $A$ site does not result in M-I transition at near room temperature. Reports on heavy rare earth doped manganite are scarce in literature and it was thought that $\mathrm{Gd}$ is a candidate cation that can be incorporated on the $A$ site. A composition, $\mathrm{Gd}_{0.7} \mathrm{Sr}_{0.3} \mathrm{MnO}_{3}$, was chosen for this study because this composition exhibits the highest magnetoresistance ratio. $\mathrm{Gd}$ is chosen as the rare earth ion because of the significant decrease of $\mathrm{Mn}-\mathrm{O}-\mathrm{Mn}$ bond angle and as a consequence it suppresses the $\mathrm{M}-\mathrm{I}$ transition temperature. Thus evaluation of conducting properties in the low temperature regime will help probe the mechanism of conduction in these compounds. $\mathrm{Gd}_{0.7} \mathrm{Sr}_{0.3} \mathrm{MnO}_{3}$ is prepared by wet solid state reaction method. The electrical conductivity studies in the low temperature regime will enable to propose a plausible mechanism for conduction in Gd based manganites. The conduction mechanism in the paramagnetic phase is a matter of controversy as different schools of thought propose different models for conduction. For example, data on certain compounds were fitted with purely activated law (Kusters et al 1989; Hundley et al 1995; Xiong et al 1996). While some authors proposed small polaron hopping conduction mechanism (SPH) over extended temperature ranges (Holstein 1959; Jakob et al 1988; Snyder et al 1996; Yeh et al 1996, 1997). At the same time, Mott and Davies used the variable range hopping mechanism (VRH) for the whole temperature range (Mott 1990; Mott and Davies 1971). Coey et al (1999) and Sun et al (2000) used VRH in a temperature interval $T_{\mathrm{C}}<T<T_{\mathrm{D}} / 2$ and small polaron hopping mechanism for temperature $T>T_{\mathrm{D}} / 2$, where $T_{\mathrm{D}}$ is the Debye temperature. The small polaron hopping mechanism appears to be most suited to manganites with smaller ionic radii rare earth ions because small polarons result from the structural distortions because of the lower ionic radii. It is becoming generally recognized that the presence of small polarons plays a key role in the peculiar transport properties of manganites, the true nature and the exact transport process of small polarons is still an open question and a matter of dispute and warrants further investigations.

\section{Experimental}

The polycrystalline samples of $\mathrm{Gd}_{0.7} \mathrm{Sr}_{0.3} \mathrm{MnO}_{3}$ were prepared using wet solid state reaction methods. Stochiometric amounts of $\mathrm{Gd}_{2} \mathrm{O}_{3}, \mathrm{SrCO}_{3}$ and $\mathrm{MnO}_{2}$ were mixed in concentrated $\mathrm{HNO}_{3}$. The solution was heated, boiling off the excess $\mathrm{HNO}_{3}$. This precursor was calcined in air at $900^{\circ} \mathrm{C}$ for several hours. The remaining black powder was pressed into pellets and sintered at $1200^{\circ} \mathrm{C}$ in air for several days (Sagar et al 2009, 2010). The sample was analysed by means of X-ray Powder Diffractometer (Rigaku Dmax C) using $\mathrm{Cu}-\mathrm{K} \alpha$ radiation $(\lambda=1.5414 \AA)$. The low temperature d.c. conductivity measurements were carried out by using source measuring unit and by cooling the sample using CTI-CRYOGENICS Model 22C cryodyne cryocooler.
This system uses helium as the refrigerant and can be interfaced with many instruments that requires cryogenic temperatures. The pressure maintained in the compressor was 400 PSIG/2758 kPa. The temperature was controlled using Lake Shore Model 321 Autotuning Temperature Controller, which had a stability of $\pm 0 \cdot 1 \mathrm{~K}$. The pressure inside the cryocooler was maintained at $10^{-5} \mathrm{mB}$ with the help of INDO VISION Vacuum Pumping System Model VPS-100. The d.c. conductivity system is fully automated by using the software called ICS.

\section{Results and discussion}

The X-ray powder diffraction pattern obtained for $\mathrm{Gd}_{0.7} \mathrm{Sr}_{0.3} \mathrm{MnO}_{3}$ is depicted in figure 1 . The pattern is characteristic of a single phasic pristine compound. The $d$-values are in good agreement with earlier reports and the structure corresponds to orthorhombically distorted perovskite (space group, Pbnm) (Asamitsu et al 1995; Garcia-Landa et al 1998). It may be noted that there are no evidences for any impurity phases in this compound. The variation of resistivity with temperature is plotted and is depicted in figure 2 . It is evident from the figure that there is marked slope change at $40 \mathrm{~K}$. This is indicative of a metal to insulator transition. In the case of manganites the resistivity increases with increase in temperature in the metallic regime (positive slope in the resistivity vs temperature graph). But in the case of low bandwidth manganites there occurs no transition at these temperatures in the absence of a magnetic field. This is in agreement with the reported results (Garcia-Landa et al 1998). The variation of $\log (\rho / T)$ with $1 / T$ is plotted and is shown in figure 3.

In the paramagnetic regime mainly three types of mechanism have been found to be ruling the conduction process in these compounds. They are (i) thermal activation or band gap model, (ii) variable range hopping model (VRH) and (iii) small polaron hopping model (SPH).

In order to understand the conduction mechanism in the paramagnetic phase of the manganites, different graphs using

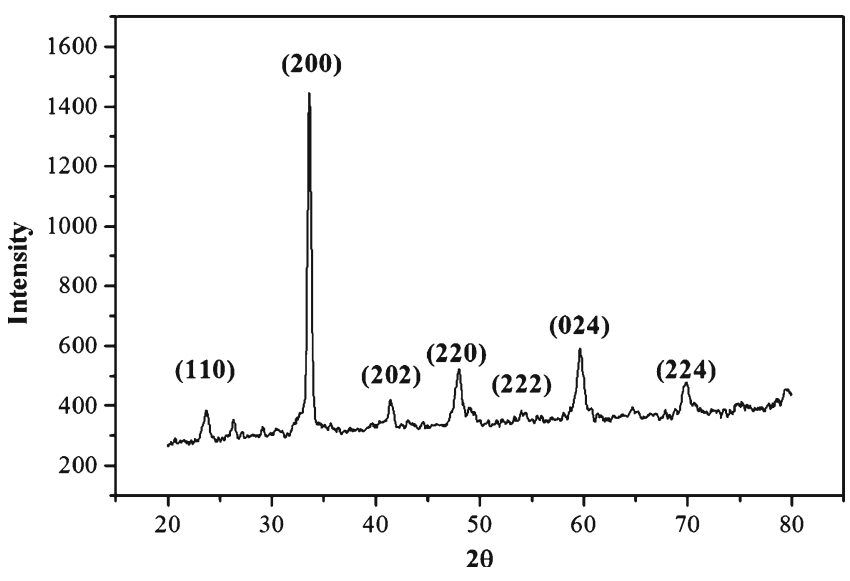

Figure 1. XRD pattern of $\mathrm{Gd}_{0.7} \mathrm{Sr}_{0.3} \mathrm{MnO}_{3}$. 


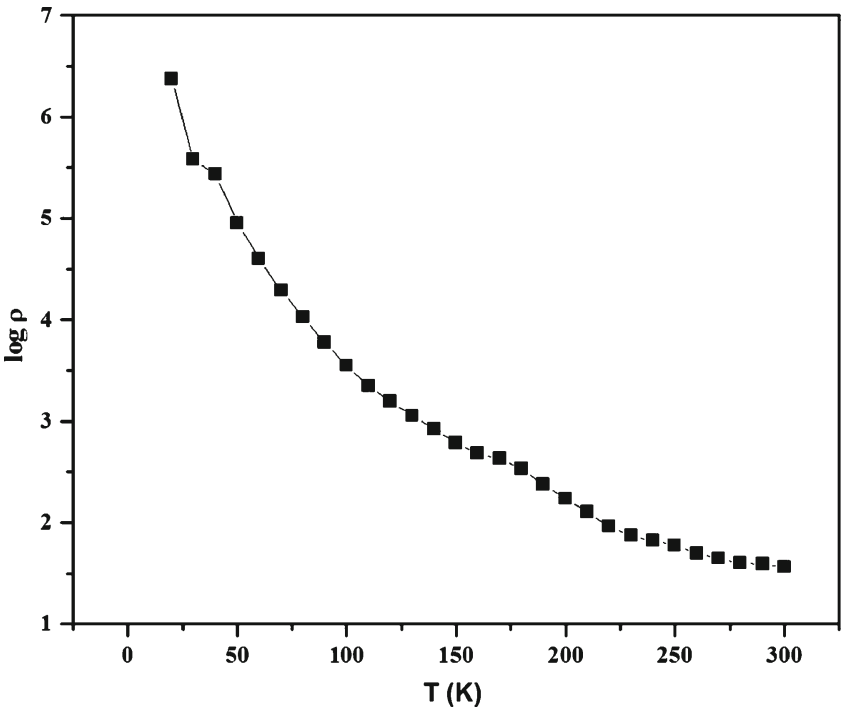

Figure 2. Variation of $\log \rho$ with temperature (lines in figure are guide to eye).

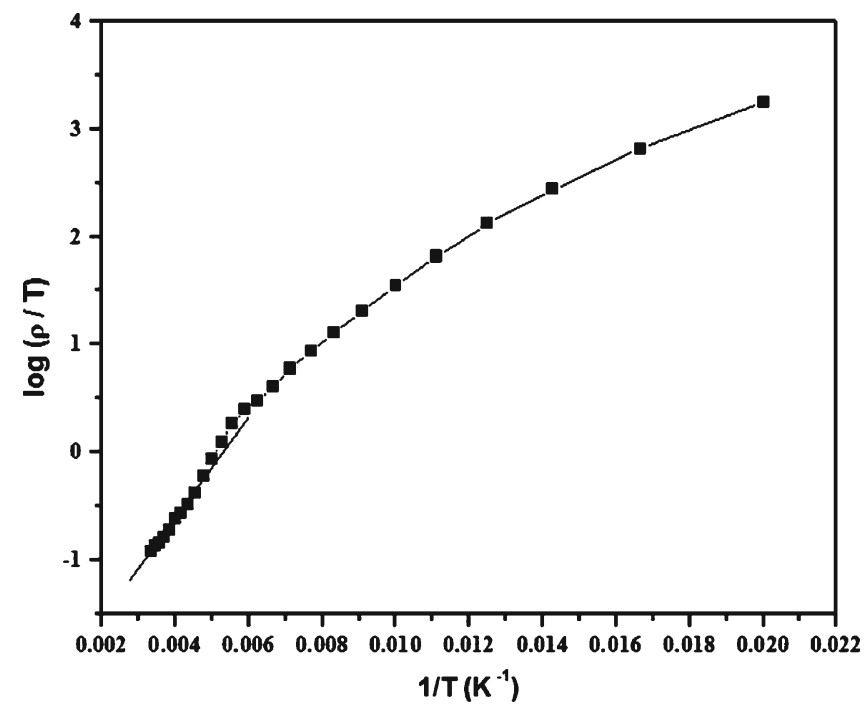

Figure 3. Variation of $\log (\rho / T)$ with $1 / T$ (lines in figure are guide to eye).

different equations are to be plotted in order to verify the veracity of that mechanism. Bandgap model is widely employed in most of the semiconductors and insulators (Kusters et al 1989; Hundley et al 1995; Xiong et al 1996). There is an energy gap between conduction band and valence band. If the thermal energy is sufficient to overcome the bandgap the electron becomes free to conduct. The expression for resistivity can be written in the following form

$$
\rho=\rho_{0} \exp \left(E_{\mathrm{A}} / k_{\mathrm{B}} T\right),
$$

where $T$ is the absolute temperature, $\rho_{0}$ the value of resistivity at infinite temperature, $E_{\mathrm{A}}$ the activation energy and $k_{\mathrm{B}}$ the Boltzmann's constant, i.e., for the bandgap model the graph must exhibit Arrhenius temperature dependence (straight line behaviour between $\log \rho$ and $1 / T$ ). However, in this case the graph is nonlinear (figure 2) and hence we conclude that the bandgap model is insufficient to explain the conduction process of this material. The second possibility is small polaron hopping (SPH) model (Holstein 1959; Jakob et al 1988; Snyder et al 1996; Yeh et al 1996, 1997). In the case of small polarons (deeply trapped electrons), the thermal energy is not sufficient to overcome the deep potential well and to hop out of its site. Then the hopping is possible by a multiphonon assisted process (Sun et al 2000). That is, the electron is activated to an intermediate state first, which is still a localized state with higher energy. Then the thermal energy acquired from the second phonon is sufficient for hopping out from the intermediate state to its nearest neighbour. The expression for resistivity is

$$
\rho=A T \exp \left(E_{\mathrm{A}} / k_{\mathrm{B}} T\right),
$$

where $T$ is absolute temperature and $E_{\mathrm{A}}$ the activation energy and $A$ a constant. The value of $A$ is given by

$$
A=\frac{k_{\mathrm{B}}}{v_{\mathrm{ph}} N e^{2} R^{2} c(1-c) \exp (2 \alpha R)},
$$

where $N$ is the number of ion sites per unit volume, $R$ the average intersite spacing, $c$ the fraction of sites occupied by polaron, $\alpha$ the electron wave function decay constant, $v_{\mathrm{ph}}$ the optical phonon frequency and $k_{\mathrm{B}}$ the Boltzmann's constant. Further, in order to check whether the conduction process obeys SPH a graph is plotted with $\log (\rho / T)$ on the $Y$-axis and $1 / T$ on the $\mathrm{X}$-axis (figure 3 ). The graph also exhibits nonlinear nature except at the starting portion (at the high temperature region). So it can be concluded that SPH model alone cannot account for the conduction process.

Now the next alternative is to check whether the VRH model can be applied to account for the observed conduction process. According to the variable range hopping (VRH) model (Viret et al 1997; von Helmholt et al 1992; De Teresa 1992, 1996; Coey et al 1995; Jaime et al 1996), if the electron is not deeply trapped (that is a large polaron) it can hop from one site to another with phonon assistance. At low temperature the thermal energy is not enough to allow electrons to hop to their nearest neighbours, but is possible to hop further to find a site with a smaller potential difference. Since the hopping range is variable, it is called variable range hopping. In three dimensional VRH model, resistivity can be expressed as

$$
\rho=\rho_{0} \exp \left(T_{0} / T\right)^{1 / 4} .
$$

Here the straight line behaviour is between $\log \rho$ and $T^{-1 / 4}$. The constant $T_{0}$ is given by

$$
T_{0}=\frac{18 \alpha^{3}}{k_{\mathrm{B}} N\left(E_{\mathrm{F}}\right)},
$$

where $\alpha$ is the electron wave function decay constant, $N$ $\left(E_{\mathrm{f}}\right)$ density of states at Fermi level. VRH theory was developed to explain electron transport in doped semiconductors, where electrons occupying hydrogenic orbitals are localized 


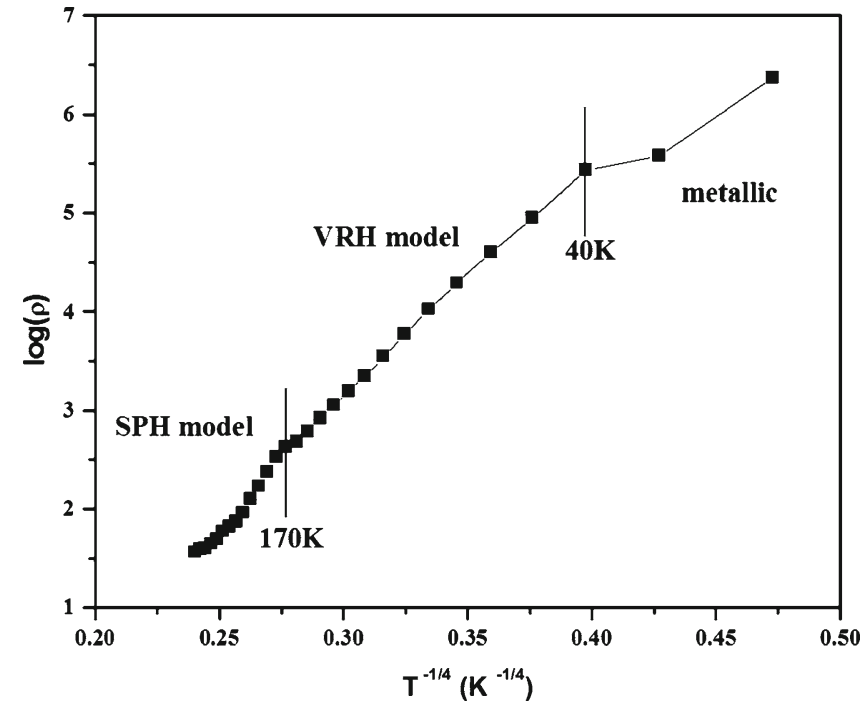

Figure 4. Variation of $\log \rho$ with $T^{-1 / 4}$ (lines in figure are guide to eye).

by potential fluctuations associated with the dopant. There is a competition between the potential difference and distance the electrons can hop (Mott 1990; Mott and Davies 1971). That is reflected in the expression for hopping rate to a site which is at distance $R$ with higher energy $\Delta E$ than the origin.

$$
\rho=\rho_{0} \exp \left(-2 \alpha R-\Delta E / k_{\mathrm{B}} T\right) .
$$

For this VRH model the graph plotted between $\log \rho$ and $T^{-1 / 4}$ should be a straight line. From the graph (figure 4) it is clear that there is perfect linear behaviour in the temperature regime of $170 \mathrm{~K}-40 \mathrm{~K}$ ( $40 \mathrm{~K}$ is the transition temperature). Thus for a wide temperature range the material obeys VRH. Above 170K the conduction is SPH assisted. From literature it is clear that some other manganites like $\mathrm{La}_{1-x} \mathrm{Ca}_{x-y} \mathrm{Na}_{y} \mathrm{MnO}_{3}$ and $\mathrm{Pr}_{0.65} \mathrm{Ca}_{0.35-x} \mathrm{Sr}_{x} \mathrm{MnO}_{3}$ also obey the $\mathrm{VRH}$ model in the temperature regime ranging from transition temperature and half of the debye temperature $\left(T_{\mathrm{c}}<T<T_{\mathrm{D}} / 2\right)$ (Bhattacharya et al 2002; Mollah et al 2004). Above the temperature, $T_{\mathrm{D}} / 2$, they obey SPH model. The activation energy for hopping mechanism, $E_{\mathrm{A}}$, can be calculated from the slope of the $\log (\rho / T)$ vs $1 / T$ graph (using (2)). The activation energy for hopping mechanism is calculated as $47 \mathrm{meV}$, which is in agreement with earlier reports (Banerjee et al 2001, 2003). Also the density of states at Fermi level $N\left(E_{\mathrm{f}}\right)$ can be calculated from the slope of $\log$ $\rho$ and $T^{-1 / 4}$ graph (using (4) and (5)). The density of states at Fermi level for the Gd based manganite is found to be $7.92 \times$ $10^{27} \mathrm{eV}^{-1} \mathrm{~m}^{-3}$ which is in good agreement with the earlier reported one (Bhattacharya et al 2002).

\section{Conclusions}

Single phasic pristine $\mathrm{Gd}_{0.7} \mathrm{Sr}_{0.3} \mathrm{MnO}_{3}$ compound was prepared by a wet chemical method. The low temperature d.c. conductivity measurements in the temperature regime $20 \mathrm{~K}-$ $300 \mathrm{~K}$ were carried out. The data thus obtained were fitted systematically to check the dominance of one particular mechanism over another viz. bandgap models, SPH and VRH. On fitting these conductivity data it has been found that the density of states at Fermi level for the Gd based manganite is $7.92 \times 10^{27} \mathrm{eV}^{-1} \mathrm{~m}^{-3}$. This is in good agreement with that of reported values. The activation energy for hopping conduction is observed to be $47 \mathrm{meV}$. It was possible to establish that the conduction in the paramagnetic phase of the $\mathrm{Gd}$ based manganite follow VRH model in the temperature range $40 \mathrm{~K}-170 \mathrm{~K}$. Below that temperature the material is ferromagnetic and metallic. Above $170 \mathrm{~K}$ the conductivity mechanism is $\mathrm{SPH}$.

\section{Acknowledgement}

One of the authors (SS) thanks the Council of Scientific and Industrial Research, New Delhi, for providing financial assistance.

\section{References}

Asamitsu A, Moritorno Y, Tomioka Y, Arima T and Tokura Y 1995 Nature 373407

Balcells L et al 1996 Appl. Phys. Lett. 691486

Banerjee A, Pal S and Chaudhuri B K 2001 J. Chem. Phys. 1151550

Banerjee A, Bhattacharya S, Mollah S, Sakata H, Yang H D and Chaudhuri B K 2003 Phys. Rev. B68 186401

Bhattacharya S, Banerjee A, Pal S, Chatterjee P, Mukherjee R K and Chaudhuri B K 2002 J. Phys. Condens. Matter 14 10221

Coey J M D and Viret M 1999 Adv. Phys. 48167

Coey J M D, Viret M, Ranno L and Ounadjela K 1995 Phys. Rev. Lett. 753910

De Teresa J M et al 1992 Nature 386256

De Teresa J M et al 1996 Phys. Rev. B54 1187

Garcia-Landa B et al 1998 J. Appl. Phys. 837664

Goyal A et al 1997 Appl. Phys. Lett. 712535

Holstein T 1959 Ann. Phys. 8325

Hundley M F et al 1995 Appl. Phys. Lett. 67860

Hwang H Y, Cheong S W and Batlogg B 1996 Appl. Phys. Lett. 68 3494

Jaime M et al 1996 Appl. Phys. Lett. 681576

Jakob G, Westerburg W, Martin F and Adrian H 1988 Phys. Rev. B58 14966

Kusters R M et al 1989 Physica B155 362

Lawler J F, Coey J M D, Lunney J G and Skumryev V 1996 J. Phys.: Condens. Matter 810737

Mahendiran R et al 1996 Phys. Rev. B53 3348

Mollah S, Huang H L, Yang H D, Pal S, Taran S and Chaudhuri B K 2004 J. Magn. Magn. Mater. 284383

Morimoto Y, Asamitsu A, Kuwahara H and Tokura Y 1996 Nature 380141

Mott N F 1990 Metal-insulator transitions (London: Taylor and Francis)

Mott N F and Davies E A 1971 Electronic processes in noncrystalline materials (Oxford University Press) 
Pedersen L A and Libby W F 1972 Science 1761355

Pollert E, Krupicka S and Kuzmicova E 1982 J. Phys. Chem. Solids 431137

Sagar S, Joy P A and Anantharaman M R 2009 Ferroelectrics 39213

Sagar S, Ganesan V, Joy P A, Senoy T, Liebig A, Albrecht M and Anantharaman M R 2010 EPL 9117008

Snyder G J, Hiskes R, DiCarolis S, Beasley M R and Geballe T H 1996 Phys. Rev. B53 14434

Sun Y, Xu X and Zhang Y 2000 J. Phys. Condens. Matter 12 10475
Venkatesan T, Rajeswari M, Dong Z W, Ogale S B and Ramesh R 1998 Philos. Trans. Roy. Soc. A356 1661

Viret M, Ranno L and Coey J M D 1997 Phys. Rev. B55 8067

von Helmholt R, Haupt L, Baïrner K and Sondermann U 1992 Solid State Commun. 82693

Xiong G C et al 1996 Solid State Commun. 97599

Yeh N C, Vasquez R, Beam D, Fu C C, Huynh H and Beach G 1996 J. Phys.: Condens. Matter 93713

Yeh N C et al 1997 J. Appl. Phys. 815499

Zener C 1951 Phys. Rev. 81440

Zhao G M, Conder K, Keller H and Muller K A 1996 Nature 381676 